\title{
Evaluación de la Producción de Biodiesel utilizando un Reactor de Película Líquida Descendente, empacado con Membranas de Fibra Hueca
}

\author{
N. Bello Yaya ${ }^{1}$, L. D. Carreño Pineda ${ }^{1}$, P. C. Narváez Rincón ${ }^{1}$, J. G. Cadavid Estrada ${ }^{1}$, A. C. Habert ${ }^{2}$ \\ ${ }^{1}$ Grupo de Procesos Químicos y Bioquímicos, Departamento de Ingeniería Química. Facultad de \\ Ingeniería, Universidad Nacional de Colombia, Bogotá, Colombia \\ ${ }^{2}$ Laboratório de Processos de Separação com Membranas e Polímeros, COPPE,Universidade Federal \\ do Rio de Janeiro, Rio de Janeiro, Brasil \\ E-mail para contato: pcnarvaezr@unal.edu.co
}

\begin{abstract}
RESUMO - Una de las principales tecnologías estudiadas para la producción de biodiésel por procesos intensificados es el uso de membranas. Diversos investigadores han realizado evaluaciones de su desempeño en la separación y purificación de los productos, especialmente en la remoción del glicerol libre y ligado, para la reacción - separación simultáneas y como soporte de catalizadores heterogéneos. En este trabajo se presentan resultados experimentales de permeación para un proceso continuo de producción de biodiésel en un reactor de película líquida descendente (FFR) usando un empaque semiestructurado que incluye membranas de fibra hueca de polietersulfona (PES). En este reactor, la metanólisis y la separación del glicerol ocurren simultáneamente, desplazando la reacción de equilibrio hacia los productos e incrementando la conversión del aceite y su rendimiento hacia ésteres metílicos, en comparación con el reactor FFR sin membranas. Un reactor a escala banco tipo FFR fue diseñado, construido y probado. El flujo de permeado de cada componente individual y de la mezcla reactiva, se determinaron para la configuración del reactor, en las condiciones de reacción. Los resultados muestran que éste tipo de empaque es adecuado para llevar a cabo la reacción-separación simultánea, con una buena selectividad a la permeación de glicerol. Sin embargo la relación molar metanol - aceite debe incrementarse para compensar el metanol que permea la membrana.
\end{abstract}

\section{INTRODUCCIÓN}

El biodiesel se ha posicionado como una de las fuentes de energía renovables de mayor interés en la última década. Según el Global Status Report (2013), la producción mundial alcanzó los 22.500 millones de litros en 2012 y, según las proyecciones de la Organización para la Cooperación Económica y el Desarrollo de las Naciones Unidas, en los próximos años seguirá aumentando (OECD, 2013). Éste biocombustible es una mezcla de ésteres metílicos de ácidos grasos, obtenida a partir de la transformación química de materias primas renovables de origen natural, específicamente aceites y grasas. Puede emplearse en motores de ignición por compresión, mezclado o no, con el diésel derivado del petróleo (Narváez et. al, 2004; Cadavid, 2012). 


\section{9 a 22 de outubro de 2014 \\ Florianópolis/SC}

Los procesos convencionales para la producción de biodiésel emplean catalizadores homogéneos, en procesos por lotes o continuos, donde tanto la reacción como las etapas de separación, pueden causar cuellos de botella, aumentando los costos operativos y limitando la viabilidad económica del proceso (Kiss et. al, 2012). Actualmente, existen varias investigaciones que buscan mejorar las tecnologías para la fabricación del biodiésel, optimizando la etapa de reacción (transesterificación), y aumentando la eficiencia de las etapas de separación y purificación del producto principal, con un menor consumo de energía (Kiss et. al, 2012; Atadashi et. al, 2011). Entre las principales tecnologías desarrolladas están el uso de reactores diferentes a los convencionales, la implementación de etapas de reacción y separación simultánea (destilación, extracción y adsorción reactiva), procesos en condiciones supercríticas y tecnología de membranas (Kiss et. al, 2012; Atadashi et. al, 2011; Shuit et. al, 2012).

Narvaez y colaboradores (2009), llevaron a cabo la producción de biodiésel en un reactor de película líquida descendente, con el fin de superar las restricciones de transferencia de masa que tiene el proceso convencional y facilitar la posterior separación de los productos. Así mismo Cadavid y colaboradores (2013), obtuvieron biodiésel con las especificaciones de la normatividad vigente, en un reactor de película líquida operado en co-corriente, realizando varias etapas de reacción - separación, debido a la limitación que presenta el equilibrio químico.

El uso de la tecnología de membranas aprovecha sus propiedades, como resistencia al estrés térmico, gran área superficial disponible por unidad de volumen, alta selectividad, y la capacidad de controlar el contacto de los componentes entre las dos fases, como una alternativa eficiente y económica, para superar las limitaciones del equilibrio químico, ya que permite la separación simultánea de uno de los productos, desplazando el equilibrio químico (Shuit et. al, 2012). Así mismo, las membranas de fibra hueca de UF (ultra filtración) se ajustan conceptual y físicamente a la configuración del reactor de película líquida descendente.

El presente documento muestra los resultados obtenidos en un reactor de película líquida descendente a escala banco, con capacidad de diseño de $5 \mathrm{~kg} / \mathrm{h}$ de aceite, operado en co-corriente, utilizando empaque semi-estructurado que incluye membranas de fibra hueca de PES. La evaluación del flujo de permeado de cada componente individual y de la mezcla reactiva, se llevó a cabo variando la presión de operación a una temperatura de reacción de $50{ }^{\circ} \mathrm{C}$. La composición de las corrientes de salida se determinó utilizando métodos gravimétricos y cromatografía de gases.

\section{MATERIALES Y METODOS}

\subsection{Materiales}

En esta investigación se usó metanol grado industrial, suministrado por QUIDISCOL (Bogotá, Colombia); aceite de Jatropha crudo, extraído a partir de las semillas específicamente para este trabajo; hidróxido de sodio grado analítico (pureza del 98\%) proveniente de Panreac (Barcelona, España); y biodiésel de Jatropha (pureza del 97\%) producido específicamente para esta investigación. 
El módulo de empaque semi-estructurado se fabricó con hilos de acero inoxidable de $0,2 \mathrm{~mm}$ de diámetro y membranas de fibra hueca para ultrafiltración de PES, suministradas por PAM Membranas (Rio de Janeiro, Brasil).

\subsection{Métodos}

Los experimentos se llevaron a cabo en un reactor de película líquida descendente, como el que se muestra en la Figura 1, con las condiciones de operación descritas en la Tabla 1.

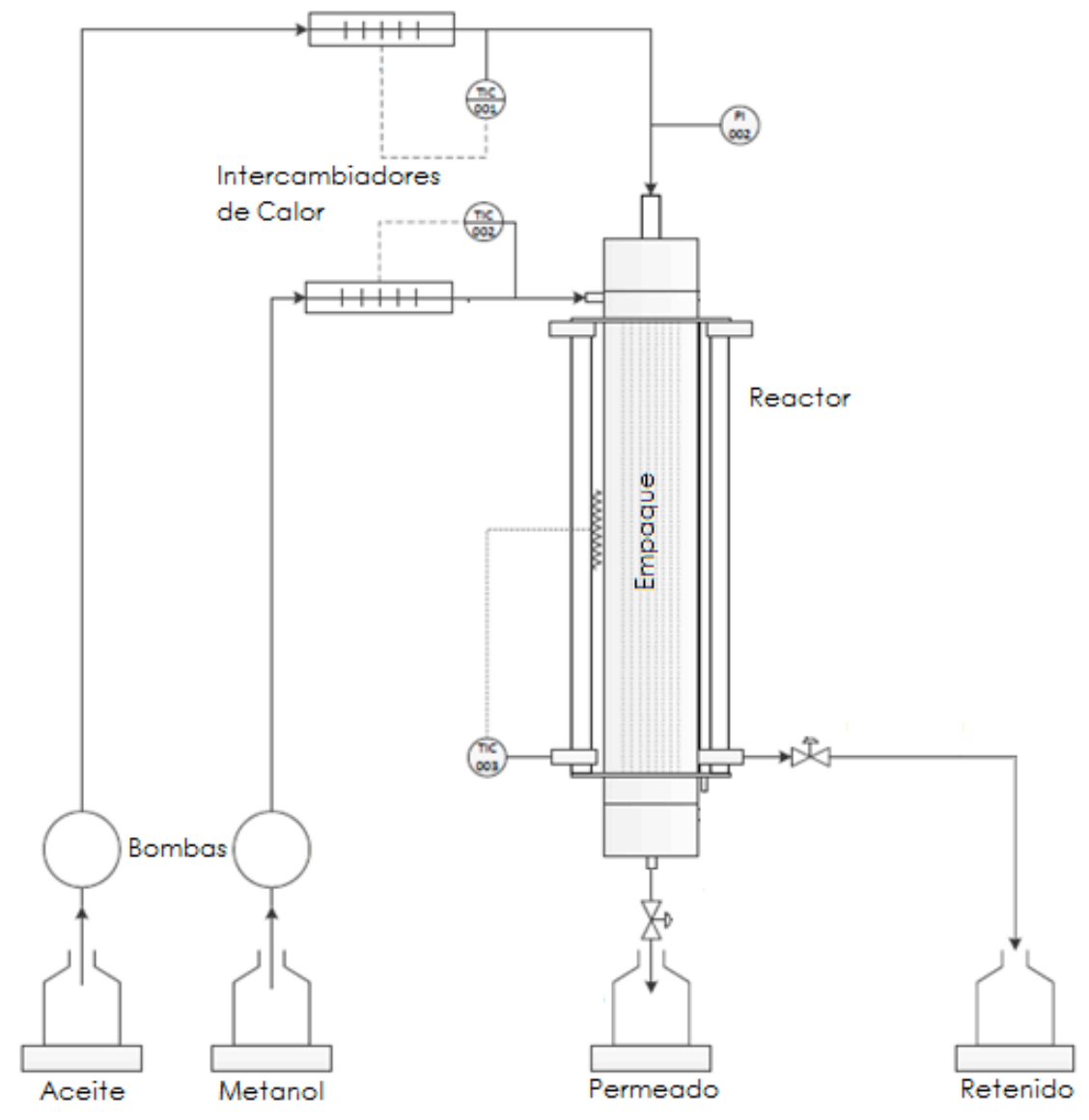

Figura 1 - Reactor de película líquida descendente para producción de biodiésel, modificado para el uso con membranas de fibra hueca.

La determinación de la permeabilidad para las sustancias puras (metanol, aceite de Jatropha, glicerol y biodiésel de Jatropha), las mezclas glicerol-metanol (25\%, 50\% y $75 \% \mathrm{p} / \mathrm{p}$ de glicerol) y la mezcla reactiva, se realizó mediante el seguimiento a la masa de permeado durante al menos 100 min. Durante este tiempo el sistema alcanzó un valor promedio estable para el flujo de permeado, y con éste valor se realizaron los cálculos de permeabilidad. Cada experimento se realizó por duplicado, variando la presión según lo descrito en la tabla 1. 
Para los experimentos con la mezcla reactiva, las muestras se tomaron en cada corriente de salida (permeado y retenido), al final de cada ensayo y se determinó su composición, utilizando métodos gravimétricos (metanol y glicerol) y cromatografía de gases (biodiésel y aceite).

Tabla 1 - Condiciones de operación para los experimentos de permeación

\begin{tabular}{|l|c|c|c|}
\hline \multicolumn{1}{|c|}{ Experimento } & $\begin{array}{c}\text { Temperatura } \\
{\left[{ }^{\circ} \mathrm{C}\right]}\end{array}$ & $\begin{array}{c}\text { Caída de } \\
\text { presión } \\
\text { Presión } \\
{[\text { bar }]}\end{array}$ & $\begin{array}{c}\text { Área activa } \\
\text { membrana } \\
{\left[\mathrm{m}^{2}\right]}\end{array}$ \\
\hline Sustancias puras & 50 & 1 & \\
\hline Mezclas glicerol - metanol & 50 & 3 & 0,026 \\
& & 4 & \\
\hline Mezcla cuaternaria & & 0,00 & \\
& 50 & 0,17 & 0,038 \\
& & 0,34 & \\
\hline
\end{tabular}

\section{RESULTADOS Y DISCUSIÓN}

\subsection{Permeación de sustancias puras y mezclas glicerol - metanol}

El flux a través de una membrana, puede describirse de manera simple por medio de la ley de Poiseuille modificada (Ecuación 1), que relaciona la porosidad $(\varepsilon)$, el diámetro de poro (d), la longitud de poro $\left({ }^{\ell}\right)$, la viscosidad del permeado $(\mu)$ y la diferencia de presión $(\Delta p)$ con el flux volumétrico.

$$
F \Delta p^{-1}=\frac{1}{32} \varepsilon d^{2} \ell^{-1} \mu^{-1}
$$

La figura 2, muestra los coeficientes de permeabilidad de cada sustancia pura y de las mezclas glicerol - metanol, calculados a partir de los datos experimentales. Se incluyen los valores de la viscosidad para cada tipo de sustancia evaluada, con el fin de observar la relación que tiene ésta propiedad con el coeficiente de permeabilidad. De acuerdo con la ecuación 1, ésta relación es inversa, lo cual también se puede observar en los resultados obtenidos.

El objetivo del uso de membranas en el reactor tipo FFR es la separación del glicerol, para desplazar el equilibrio químico en la reacción de metanólisis, y así incrementar el rendimiento hacia ésteres metílicos. Sin embargo los resultados obtenidos muestran que la permeación del glicerol se favorece cuando se encuentra mezclado con metanol en una composición superior al 50\% p/p. Esta observación es importante porque determina que la relación molar metanol - aceite que se use en los 
ensayos de reacción química, debe garantizar la cantidad de metanol suficiente para favorecer la permeación del glicerol, sin que se afecte de manera considerable el progreso de la reacción de metanólisis.

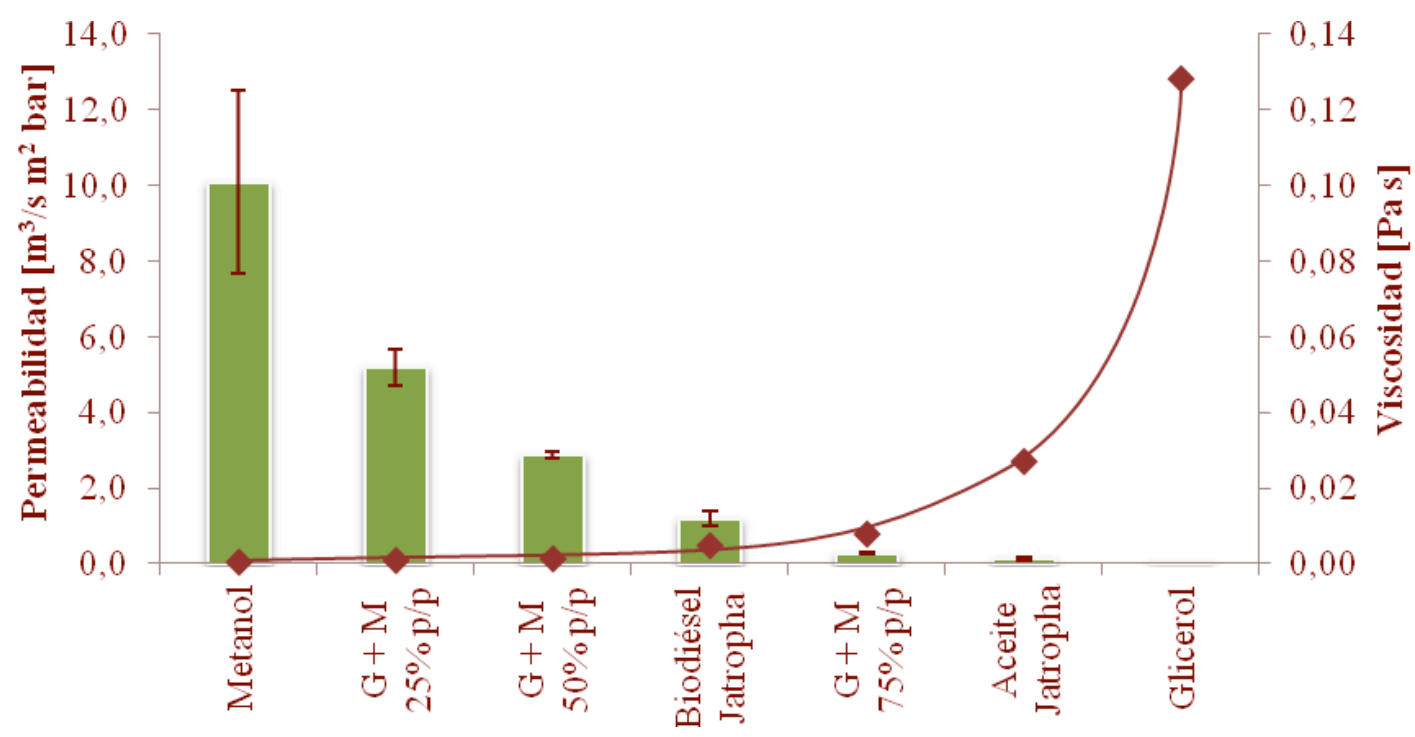

Figura 2 - Permeabilidad (barras) y viscosidad dinámica (rombos) para las sustancias puras y las mezclas glicerol - metanol. Temperatura $50^{\circ} \mathrm{C}$.

\subsection{Permeación de la mezcla cuaternaria}

La figura 4 muestra la composición de las corrientes de alimento, permeado y retenido para los experimentos realizados con la mezcla cuaternaria.

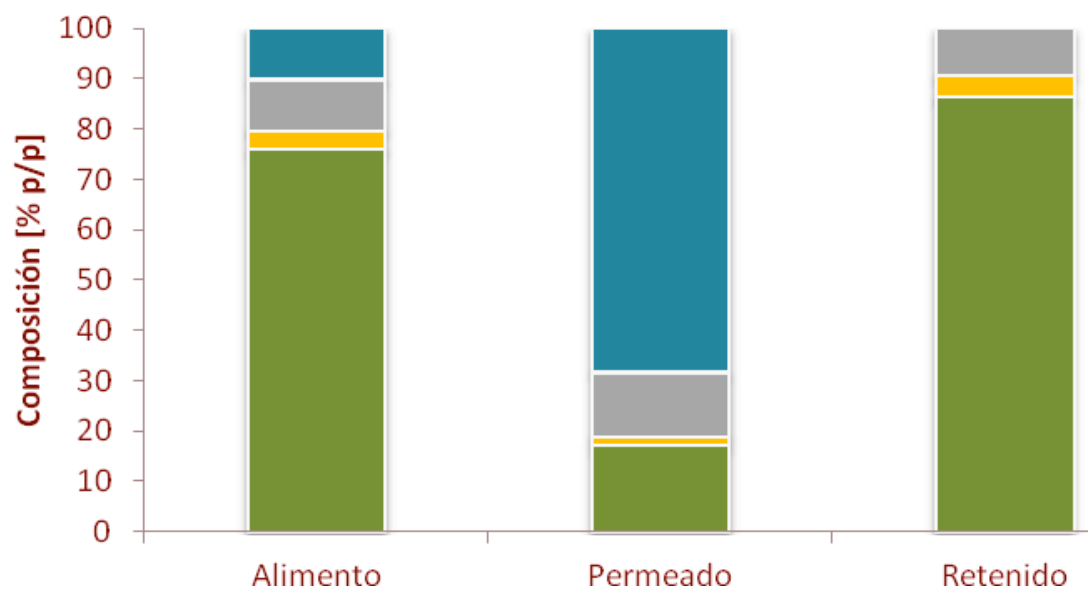

Figura 4 - Composición de las corrientes en los experimentos con la mezcla cuaternaria. Presión de vacío $0,7 \mathrm{bar}$, Temperatura $50^{\circ} \mathrm{C}$. Metanol (azul), glicerol (gris), aceite de Jatropha (amarillo), biodiésel (verde) 


\section{9 a 22 de outubro de 2014 \\ Florianópolis/SC}

La composición del permeado corresponde en su mayoría a metanol (68\%) y glicerol (13\%), lo cual se esperaba a partir del coeficiente de permeabilidad calculado en los ensayos para las sustancias puras. En cuanto al retenido es interesante observar que no se encontró metanol y la mayor composición corresponde a biodiésel (86\%). El 20\% del glicerol alimentado se permeó a través de la membrana, pero no es claro si se limitó su permeabilidad por la falta de metanol, o por la evaporación del mismo durante el proceso. Reforzando de nuevo la idea que se debe alimentar metanol suficiente para facilitar la separación (permeación) del glicerol.

\section{CONCLUSIONES}

Los resultados obtenidos muestran que la permeabilidad selectiva del glicerol, depende de la viscosidad de la mezcla glicerol - metanol, encontrando mayores coeficientes de permeabilidad para mezclas con cantidades de glicerol por debajo de $50 \%$ p/p. Esta condición limita la relación molar metanol - aceite que se debe alimentar en los ensayos con reacción química, ya que ésta debe garantizar la disponibilidad de metanol suficiente para favorecer la permeación del glicerol, sin que se afecte notablemente el progreso de la reacción de metanólisis. Sin embargo es posible la separación del glicerol y por tanto el desplazamiento de la reacción de metanólisis, con el respectivo incremento en la conversión y el rendimiento.

\section{REFERENCIAS}

Atadashi, I. M. Aroua, M. K. Abdul Aziz, a. R. Sulaiman, N. M. N. Membrane biodiesel production and refining technology: A critical review. Renewable and Sustainable Energy Reviews, 15(9), 5051-5062, 2011.

Cadavid, J. G. Estudio del proceso de producción de biodiésel mediante extracción reactiva. Tesis Doctoral en Ingeniería Química, Universidad Nacional de Colombia, 2012.

Cadavid, J. G. Godoy-Silva, R.D. Narváez, P.C. Camargo, R. D. Fonteix, C. Biodiesel production in a counter-current reactive extraction column: Modelling, parametric identification and optimisation. Chemical Engineering Journal, 228, 717-723, 2013.

Kiss, A. Bildea, C. S. A review of biodiesel production by integrated reactive separation technologies. Journal of Chemical Technology \& Biotechnology, 87(7), 861-879, 2012.

Narváez, P. C. Sánchez, F. J. Godoy-Silva, R. D. Continuous methanolysis of palm oil using a liquid-liquid film reactor, Journal of American Oil Chemists's Society, 86, 343-352, 2009.

Narváez, P. C. Sánchez, F. J. Torres, J. A. Ponce de León, F. Producción de ésteres metílicos de ácidos grasos: variables asociadas al proceso de transformación. Revista Ingeniería e Investigación, (55), 41-50, 2004. 
Shuit, S. H. Ong, Y. T. Lee, K. T. Subhash, B. Tan, S. H. Membrane technology as a promising alternative in biodiesel production: a review. Biotechnology Advances, 30(6), 1364-80, 2012.

REN21. Renewables 2013 - Global Status Report. Disponible en internet formato PDF en: http://www.ren21.net/Portals/0/documents/Resources/GSR/2013/GSR2013_lowres.pdf. Consultado 08/07/2013.

The Organisation for Economic Co-operation and Development - OECD. Biofuels - OECD FAO Agricultural Outlook 2013 - 2022. Disponible en internet formato HTM, en: http://www.oecd.org/site/oecd-faoagriculturaloutlook/biofuels.htm. Consultado 26/11/2013. 